Geopolítica(s) Revista de estudios sobre espacio y poder ISSN: 2172-3958

\title{
Reconfiguraciones del sistema mundial: nuevos imaginarios y actores sociales
}

Heriberto Cairo y Breno Bringel (Eds.) (2019) Critical Geopolitics and Regional (Re)configurations. Interregionalism and Transnationalism Between Latin America and Europe. Londres: Routledge, 232 pp. ISBN: 978-1-138-61533-5.

Profundizar en las vinculaciones entre América Latina y Europa permite aproximarse a los intersticios del interregionalismo y el transnacionalismo; no obstante, considerar ambas entidades supone afirmar que han experimentado cambios, tanto a nivel interno como en las relaciones que mantienen entre sí, y que por tanto continúan disputando con otras construcciones e imaginarios regionales que los desafían (pp.14-15). Bajo esta premisa, los profesores Heriberto Cairo (Universidad Complutense de Madrid) y Breno Bringel (Universidade do Estado do Rio de Janeiro) han publicado en 2019 un volumen colaborativo que aborda las interacciones entre dos regiones con conexión de larga data y cierta afinidad cultural. Se trata de una obra que viene a contribuir, desde la geopolítica crítica, a la literatura relacionada con las configuraciones regionales tratando de rebasar la clásica perspectiva interestatal.

Exceder las interpretaciones mayoritarias en las ciencias sociales supone abordar las profundas transformaciones acontecidas en las últimas décadas para considerar una multiplicidad de actores e imaginarios que, sin olvidar el papel de relieve que continúa teniendo el Estado, han proliferado en ambas geografías. La obra destacará entonces la dinámica y las relaciones interregionales desde una mirada dialéctica y multidisciplinar, ubicando el foco en la cooperación y el desarrollo internacional, las políticas globales, las fronteras, la desigualdad y los movimientos sociales. Estos actores, puestos bajo la lupa de la investigación social, arrojan como resultado una serie de vínculos que configuran un nuevo campo transnacional donde se desafían las territorialidades tradicionales.

La obra, que cuenta con la contribución de quince autores de amplia experiencia académica, tiene por objeto general aportar una nueva mirada sobre las relaciones euro-latinoamericanas desde las perspectivas emergentes de los estudios globales y transnacionales, superando la perspectiva que incide únicamente en la interacción entre Estados, cierta idea de una modernidad incompleta en América Latina y una visión esencialmente negativa de las relaciones entre esta y Europa (pp.7-8). Segmentado en 11 capítulos, cuenta con un prefacio a cargo de Walter Mignolo donde el autor trata de marcar las limitaciones con las que, frecuentemente, el investigador de las ciencias sociales se encuentra a la hora de abordar la conexión entre am- 
bos continentes, advirtiendo del eje de modernidad/colonialidad que se ha erigido en epicentro de su desarrollo teórico y se convierte aquí en punto de partida epistemológico.

El volumen clasifica su contenido en tres bloques temáticos bien definidos: el primero de ellos trata las dos regiones que nuclean el texto -Europa y América Latina - en el sistema-mundo contemporáneo, tomando en cuenta cuestiones como la imperialidad, la dominación y la cooperación. El geógrafo David Slater abre este primer apartado abordando el intervencionismo y la política de la "invasividad" que los países de Occidente, y esencialmente Estados Unidos, han llevado a cabo con el objetivo que asegurar su dominación en el sistema mundo actual. Distinguiendo entre imperialismo e "imperialidad" (imperiality), alude al privilegio de ser percibido como actor legítimo para ejercer dicha invasividad que revela una relación asimétrica entre las sociedades imperiales y las imperializadas (p.27). Gracias a un completo despliegue de formas de poder y estrategias, se conforma una especie de "sentido común" que apunta a un sentimiento de supremacía sedimentado y justificado en una idea de Occidente como proveedor de valores como la democracia, la civilización, la modernidad y la libertad. Señala Slater que, añadido a los significados movilizados en el intervencionismo geopolítico - el deseo, la voluntad política, las capacidades y la justificación - es posible encontrar también resistencias a una política imperial, la de Estados Unidos, que ha marcado trascendentalmente la trayectoria de la región latinoamericana.

Por su parte, José Maurício Domingues profundiza en la idea de hegemonía del liberalismo en las formas de dominación global explorando su versión más reciente: el social liberalismo, expresado en las serialidades cerradas y en las políticas sectorializadas. Según señala el autor, el social liberalismo ha conjugado con sutileza la economía con la biopolítica particularmente en regiones donde el problema de la pobreza se presenta notablemente. De esta manera, el carácter individualista del liberalismo, agitando la bandera del emprendimiento, se ha inclinado por convertir derechos universales en un tratamiento diferenciado de los sectores pauperizados, de colectividades específicas con identidades particulares y cada vez más cerradas que ha alcanzado la escala global. Ante un panorama de constante expansión del particularismo, Domingues propone el planteamiento de políticas homogeneizadoras que tengan al ciudadano-trabajador como sujeto de derechos universales, avanzando hacia una solidaridad compleja (p.57) que tome en cuenta la multiplicidad de colectividades con aspiraciones emancipadoras sin dejarse capturar por la estrategia fragmentadora.

Heriberto Cairo se adentra específicamente en el interregionalismo eurolatinoamericano, ubicando el escenario de las relaciones entre ambos entes geopolíticos en el orden mundial de la posguerra fría, cuyas consecuencias han derivado en altos niveles de globalización y militarización y una multipolaridad que ha complicado la definición de hegemonía a partir del declive de Estados Unidos. Realizando una reconstrucción de las cumbres celebradas entre las dos regiones, encuentra que desde finales del pasado siglo existe un objetivo de establecer una "asociación estratégica birregional", que marcaría el surgimiento del mencionado interregionalismo y que se sostendrá sobre la idea de un patrimonio cultural común, una valoración positiva de la globalización y el impulso de relaciones horizontales como estrategia de competencia en el mundo globalizado. De forma similar, la Comuni- 
dad Iberoamericana de Naciones tiene como embrión el interés de España por erigirse en epicentro del encuentro entre Europa y los países latinoamericanos, una suerte de avanzadilla que posteriormente la Unión Europea aprovechó para intensificar la estrategia interregional. La experiencia de las últimas décadas ha convertido así a Europa en exponente del soft power en contraste con la táctica militarizada de Estados Unidos.

El segundo bloque se aproxima a los imaginarios geopolíticos y los órdenes socio-territoriales que permanecen en las dos regiones a tratar, proyectando una perspectiva desde el tratamiento de la formación de los Estados, los replanteamientos de Europa a partir de sus territorios "olvidados", las narrativas relacionadas con la "baja política" o las fronteras y sus significados en la geopolítica moderna. Para Pedro Borba se trata aquí de discutir la sociología histórica de los Estados modernos de América Latina, alejándose nuevamente de los enfoques desarrollistas y eurocéntricos. Para ello da cuenta del colonialismo como proceso interregional de más profundidad y longevidad y propone cerrar tres brechas: entre la teoría social y los llamados estudios de área, entre la sociología histórica y la historiografía global, y entre la literatura poscolonial predominantemente anglófona y la tradición crítica latinoamericana (p.79). La idea que subyace a lo largo del capítulo es que, en la producción académica, a menudo se ha ubicado Europa como modelo de la formación del Estado y la modernidad, relegando a América Latina al papel de "desviación". La solución, según Borba, pasaría por desplazar los procesos de formación de los Estados como resultado de la mera causalidad endógena a la perspectiva de un sistema interestatal moderno que implicó a ambas orillas del Atlántico; y reemplazar el difusionismo eurocéntrico por el reconocimiento de la naturaleza multicéntrica del cambio en la historia global.

Colonialismo e imperialismo son también las preocupaciones centrales en el capítulo a cargo de Manuela Boatcã, que cuestiona la noción occidentalista de "europeidad" a partir de las fronteras no reconocidas en el Atlántico y el Caribe. El continente europeo ha revelado la geopolítica y la epistemología de diferentes momentos históricos, pero la naturalización de Europa como ente con "sentido común" ha dado lugar a excepciones que, en realidad, se han producido de manera sistemática. La construcción discursiva de una noción singular de Europa depende crucialmente del silenciamiento del papel histórico de sus Estados miembros (p.100), imponiéndose como referentes aquellos que han ejercido el dominio imperial, lo cual se imprime en cierta "geografía moral" que refleja el discurso civilizador y que acaba sosteniendo una jerarquía de Europas múltiple y desigual. El ejemplo más claro que encuentra la autora son las posesiones coloniales, a las que denomina "Europas olvidadas", que nunca se incluyeron en los discursos de la modernidad y la europeidad. Pese a ello, considera Boatcã que son precisamente los territorios europeos en el Caribe los que ofrecen una perspectiva privilegiada para repensar la representación dominante de Europa. Como consecuencia, la "criollización" de Europa supone reescribir las experiencias transnacionales no europeas y replantear también sus propias fronteras.

Olivier Thomas Kramsch se imprime igualmente en el enfoque fronterizo al explorar las prácticas más mediadoras de las relaciones socio-espaciales, específicamente entre Europa y América Latina, a través de la génesis y evolución de lo que denomina "fronteras letradas" (lettered border). El autor señala aquí la estrecha 
relación entre los procesos de fronterización interiores (Estados europeos) y los antiguos frentes coloniales, extendidos a la configuración geopolítica actual, y al mismo tiempo argumenta que el carácter de los frentes está sufriendo transformaciones que inutilizan cada vez más los modelos centro-periferia. Kramsch propone ver las fronteras latinoamericanas y europeas como un "vaivén de horizontes" (p.138), una revelación de espacios que apuntan a otros y que están impregnadas también de una temporalidad dinámica y un deseo político, presentándose una constante interdependencia.

María Lois continúa adentrándose en la temática fronteriza desde los imaginarios geopolíticos, que nuevamente inciden en una diferenciación entre los centros de poder (Europa) y las periferias (América Latina), presentándose los primeros como ejemplo de libre circulación y los segundos, de fronteras como sinónimo de perpetua disputa. Siguiendo a John Agnew', Lois señala que la "imaginación geopolítica moderna", que se ha materializado en supuestos geopolíticos dominantes que redefinen constantemente el sentido común del espacio global, fomenta una jerarquía de bloques espaciales proyectados como homogéneos y que se definen según su relación con la trayectoria del bloque dominante. Partiendo de procesos superpuestos y significados complejos, la autora se propone una aproximación a los imaginarios y prácticas de articulación, superposición, negociación, alteridad o resistencia, sobre la base del inevitable encuentro que involucra la construcción de ambas regiones (p.148). La centralidad de la frontera como práctica de construcción y reproducción social y no como mero dispositivo binario es punto de partida para entender los procesos fronterizos, donde a menudo la espectacularización de las dinámicas de conflicto han opacado realidades más complejas.

El bloque también cuenta con la aportación de Rosa de la Fuente y Renato Miranda, que abordan la construcción de imaginarios espaciales —en este caso, nacionales - en contextos de crisis: una reformulación de la escena geopolítica con el fin de contrarrestar los efectos de las agencias de calificación y relanzar la proyección de un país. Para ello toman el ejemplo de la "Marca España" en la última década y la autopresentación como centro para la actividad de las empresas translatinas, fenómeno que puede estudiarse como una tendencia a la "mercantilización" del Estado con el empleo de recursos públicos para intereses privados —o "baja política" - pero también como estrategia para el establecimiento de relaciones con mayor simetría. Las nuevas narrativas, que fomentaron la idea de América Latina no solo como escenario para invertir sino también como inversor potencial, fueron instrumentalizadas para fomentar un transnacionalismo liderado por la iniciativa privada y la obtención de una mejor posición a la hora de liderar el interregionalismo entre Europa y América Latina.

El tercer y último bloque toma en cuenta la cuestión del interregionalismo a partir de las conexiones transnacionales de los movimientos sociales, que en las últimas décadas han sido capaces de poner en contacto una serie de actores a partir de solidaridades hacia luchas que no por ser localizadas han dejado de ser globales, como los ciclos de movilización feminista, el neozapatismo o el de "los indigna-

J. A. Agnew: Geopolítica: una re-visión de la política mundial, Madrid, Trama Editorial, 2005.

J. A. Agnew: "Baja" Geopolítica: agencias de calificación, la privatización de la autoridad y la nueva soberanía, Geopolítica(s). Revista de Estudios sobre Espacio y Poder, 3(2), 2012, 171-183. 
dos". La apertura corre a cargo de los coordinadores del libro, en esta ocasión para reflexionar sobre la solidaridad internacional y las redes transnacionales que tratan de cerrar la brecha entre organizaciones del Norte y el Sur Global, esencialmente entre Europa y América Latina, durante el ciclo abierto por el levantamiento zapatista de 1994 en Chiapas hasta el estallido de la crisis financiera en 2007. Para ello retoman el hilo tendido por Boaventura de Sousa Santos y su "racionalidad cosmopolita", basada en tres preceptos: la sociología de las ausencias, la sociología de la emergencia y el trabajo de traducción. Esta última permitiría la inteligibilidad mutua y fomentaría el fortalecimiento de las convergencias y sinergias de la diversidad. En un contexto iberoamericano, encontramos grupos de solidaridad internacionalista, comités de apoyo, asociaciones y otros grupos similares que se conforman como traductores privilegiados de prácticas y conocimientos. En la transición del internacionalismo clásico al "internacionalismo complejo" —o "nuevo internacionalismo solidario"-, la ruptura de la lógica binaria global-nacional atraviesa la territorialidad del Estado-nación y ha potenciado un imaginario regional de América Latina más autónomo y potencialmente emancipador que surge como espacio de enunciación contra-hegemónico donde convergen las organizaciones anti-globalización neoliberal del Norte con las del Sur, exponiendo como ejemplos los Comités de Solidaridad Internacionalista en España y Portugal y la red birregional Enlazando Alternativas.

Desde la geopolítica feminista, Almudena Cabezas y Gabriela Brochner plantean examinar la acción transnacional del movimiento de mujeres para dar cuenta de sus dimensiones regionales e interregionales, considerando actores no estatales y traspasando el poder normativo europeo. El capítulo aborda entonces el ciclo de movilizaciones acontecido entre 2014 y 2018, año de la huelga global de mujeres. Entendiendo que el compromiso político trasciende cada vez más los territorios tradicionales para conectar diversas escalas, se analiza aquí el impacto y la retroalimentación del movimiento polaco \#BlackProtest y del argentino \#NiUnaMenos como núcleo de una serie de protestas que traspasaron las fronteras y conectaron con diversos espacios geográficos, y en los que una demanda global adaptó más tarde la forma de cada particularidad regional con la voluntad de formar un frente feminista internacional y una agenda completa. Un ejemplo similar de contagio interregional fue la Red Federica Montseny, que a partir de la protesta contra la reforma legal del aborto en España en 2014, logró obtener un eco en ciudades como Buenos Aires y Montevideo; así como la Red Latinas logró poner de relieve las realidades particulares de las mujeres migrantes. Las autoras abogan así por una sistemática inclusión de la perspectiva feminista en el estudio del interregionalismo.

Retomando las solidaridades internacionales, Livia Alcantara y Breno Bringel cierran este tercer bloque analizando las conexiones transnacionales entre México y Barcelona, a partir del surgimiento del neozapatismo en la década de los noventa y ante la irrupción de un ciclo de protestas de nuevo cuño en el escenario de la crisis económica mundial. Como ya habían adelantado Cairo y Bringel, el fin del internacionalismo clásico dio paso a un nuevo horizonte alternativo que emergió de la experiencia en la Lacandona, donde el EZLN germinó un nuevo imaginario de resistencia capaz de conectar con las luchas de distintos puntos del planeta, indicando que las luchas localizadas no son necesariamente locales y desafiando la 
forma estatal contemporánea para repensar el cambio social (p.198). Con el objetivo de analizar las nuevas configuraciones de solidaridad transnacional relacionadas con el activismo social entre Europa y América Latina, se realiza un recorrido por la transición del ciclo de solidaridad zapatista al posterior ciclo de luchas de los indignados, con el foco en el activismo que enlaza Chiapas con la ciudad de Barcelona. De un primer movimiento - el neozapatista - que trabajó intensamente las redes transnacionales y un pionero ciberactivismo, de hondo calado en las redes de solidaridad en la capital catalana, en la primera década del nuevo siglo la lucha antiglobalización viró hacia espacios más tematizados, reemplazando convergencias más amplias de aspiración global. La crisis global, que impactó con especial fuerza en México y los países del sur europeo, volvió a conectar ambos escenarios con una solidaridad más coyuntural y efímera. El impulso de ambos ciclos fluyó por tanto de la revitalización del autonomismo y el contrapoder a un desafío del sistema político en un escenario de profundas desigualdades.

El epílogo redactado por Teivo Teivainen coloca el broche al hilo conductor, argumentando que los cambios de la última década, en gran parte resultado de la crisis europea, otorgan oportunidades para establecer una conexión más democrática con el Sur Global, en un intercambio de experiencias de doble sentido donde Europa deje de ejercer su poder normativo.

En definitiva, la obra coordinada por Cairo y Bringel viene a contribuir a la literatura académica de la geopolítica crítica, con el fin de entender procesos más amplios que imbrican a América Latina y a Europa en un tejido de relaciones en ambas direcciones y siempre en construcción. Una nueva mirada que brinda atención a las reconfiguraciones del sistema mundial y que aboga por una sistemática inclusión de nuevos imaginarios y actores sociales. El abordaje de una multiplicidad de arenas en las que dichas relaciones pueden manifestarse hacen de este título una plataforma para aproximarse al estudio del interregionalismo.

Elena Moreno Gabino

Facultad de Ciencias Políticas y Sociología Universidad Complutense de Madrid Email: elenamoreno.gc@gmail.com 